\title{
Curriculum deep reinforcement learning with different exploration strategies: A feasibility study on cardiac landmark detection
}

\author{
Patricio Astudillo ${ }^{1,2} \mathbb{D}^{\text {a }}$, Peter Mortier ${ }^{1}$, Matthieu De Beule ${ }^{1}$ and Francis wyffels ${ }^{2} \mathbb{D}^{\mathrm{b}}$ \\ ${ }^{1}$ FEops, Technologiepark 122, Zwijnaarde, 9052, Belgium \\ ${ }^{2}$ Department of Electronics and information systems, UGent - imec, Technologiepark 126, Zwijnaarde, 9052, Belgium
}

\begin{abstract}
Keywords: Biomedical Informatics, Cardiography, Medical Information Systems, Semi-Supervised Learning Abstract: Transcatheter aortic valve implantation (TAVI) is associated with conduction abnormalities and the mechanical interaction between the prosthesis and the atrioventricular (AV) conduction path cause these life-threatening arrhythmias. Pre-operative assessment of the location of the AV conduction path can help to understand the risk of post-TAVI conduction abnormalities. As the AV conduction path is not visible on cardiac CT, the inferior border of the membranous septum can be used as an anatomical landmark. Detecting this border automatically, accurately and efficiently would save operator time and thus benefit pre-operative planning. This preliminary study was performed to identify the feasibility of 3D landmark detection in cardiac CT images with curriculum deep Q-learning. In this study, curriculum learning was used to gradually teach an artificial agent to detect this crucial anatomical landmark from cardiac CT. This agent was equipped with a small field of view and burdened with a large action-space. Moreover, we introduced two novel action-selection strategies: $\alpha$-decay and action-dropout. We compared these two strategies to the already established $\varepsilon$-decay strategy and saw that $\alpha$-decay yielded the most accurate results. Limited computational resources were used to ensure reproducibility. In order to maximize the amount of patient data, the method was cross-validated with k-folding for all three action-selection strategies. An interoperator variability study was conducted to assess the accuracy of the method.
\end{abstract}

\section{INTRODUCTION}

Transcatheter aortic valve implantation (TAVI) has become the preferred treatment for patients with aortic stenosis at high risk for surgical aortic valve replacement (SAVR) (Smith, 2011). Recently, studies concluded that TAVI was similar to SAVR for intermediate-risk patients concerning the primary end-point of death or disabling stroke (Leon, 2016; Reardon, 2017). Very recent clinical data showed that TAVI is at least as good as SAVR in low-risk patients (Popma, 2019; Mack, 2019). Although TAVI is less-invasive than SAVR, it is associated with an increased incidence of higher grade atrioventricular (AV) block that requires permanent pacemaker implantation (Reardon, 2017). The conduction abnormalities are due to the mechanical interaction between the prosthesis and the AV conduction path located near the aortic valve. Preoperative assessment of the location of the AV conduction path and the expected contact pressure in this region can help to understand the risk of postTAVI conduction abnormalities (Rocatello, 2018; Hamdan, 2015). As the AV conduction path itself is not visible on the cardiac CT images, the inferior border of the membranous septum can be used as an anatomical landmark. Detecting the inferior border of the membranous septum can be a difficult task that dependents on operator experience, image quality, and the patient's anatomy. Detecting these points in an automatic, accurate, and efficient manner would save operator time and thus benefit pre-operative planning.

Advancements in deep learning have shown that there are supervised methods that can detect landmarks in medical images (Zheng, 2012; Zheng, 2015; Zhang, 2017; Payer, 2016; O’Neil, 2018; Al, 2018; Litjens, 2017). These methods analyse a delimited region of interest and thus share the same

a https://orcid.org/0000-0002-5503-6217

b[D https://orcid.org/0000-0002-5491-8349 
limitation because they require a region of interest annotation. In this study, we wished to overcome this limitation and analyse medical images that range from region-specific to full-body scans.

Recent studies have shown that deep reinforcement learning can be used to detect landmarks in medical images regardless of their dimensions (Ghesu, 2016; Ghesu, 2017a; Ghesu, 2017b; Alansary, 2019; Sahiner, 2019). In these studies, accurate results were obtained using a cluster of computational resources. However, the following difficulties may arise when exploring their methods. Deep reinforcement learning introduces novel hyper-parameters on top of the already existing hyper-parameters specific to deep learning and machine learning. When a cluster of computational resources is unavailable, the hyperparameter search may require a considerable amount of time. Moreover, since deep reinforcement learning is still developing, it is essential to look into mechanisms that require additional research, e.g. in the two studies (Ghesu, 2017b) and (Alansary, 2019) the authors have a different opinion on the definition of the halting signal for the artificial agent. Therefore, novel strategies should be studied and evaluated in a reproducible manner.

In this preliminary study, we aimed to identify the feasibility of 3D landmark detection in cardiac CT images with curriculum deep Q-learning. This novel method of learning allowed us to use limited computational resources and tune the hyperparameters accordingly. It also enabled us to evaluate three strategies that drive the exploring character of the artificial agent: the already established $\varepsilon$-decay strategy and two novel strategies $\alpha$-decay and action-dropout. Additionally, the agent was equipped with a larger action-space and smaller state-space when compared to the state-of-the-art. Each strategy was validated with k-folding; a crossvalidation method that allows the usage of the entire dataset for validation while maintaining a strict separation between the training and validation dataset. An inter-operator variability study was conducted to assess the accuracy of the method.

\section{METHOD}

\subsection{Patient and imaging data}

This retrospective study used the anonymised data of 278 patients from multiple centers. The mean age of this cohort was $80.7 \pm 6.6$ years, and $56 \%$ of the patients were female. The patient data consisted of volumetric cardiac CT images, which were acquired during the pre-operative planning of a TAVI procedure. Therefore, all CT images were contrast-enhanced and contained a certain degree of aortic stenosis. The dimensions of the images ranged from regional scans, centered around the aortic valve, to scans of the entire body.

An expert analysed all images and identified the inferior border of the membranous septum. Three 3D points defined this inferior border: MS1, MS2 and MS3 and were considered the ground truth in our study (Figure 1).

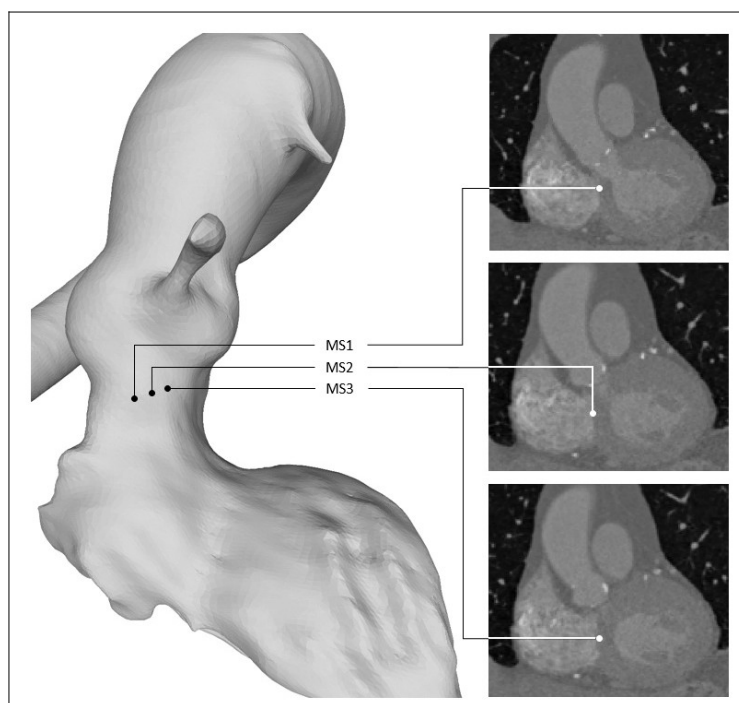

Figure 1: The 3D view (left) and the coronal view (right) of the aortic valve with the annotated ground truth points: MS1, MS2 and MS3.

The volumetric CT images were preprocessed in the following manner: first, they were resampled to obtain a homogenous dataset. Next, each image was interpolated to isotropic resolutions of 1.0, 2.0, 3.0 and $5.0 \mathrm{~mm}$ using cubic spline interpolation. Finally, all images were normalised. 


\subsection{Landmark detection with reinforcement learning}

The task of detecting landmarks in medical images with reinforcement learning can be described as follows. An artificial agent is positioned in an environment (an image) where it can walk around in search of the target landmark. Based on its current position, the agent has a limited field of view, causing it to see only a small image patch (the state). From its current state s, the agent needs to choose an action a, which will move him closer towards the target landmark. After performing that action, the agent will be at a new position in the image and thus have a new state $\mathbf{s}$ '. The reward $\mathbf{r}$ for choosing an action is the delta between the Euclidean distance from the previous- and current position to the target landmark. The agent's experience is defined as $\mathbf{s}$, a, $\mathbf{s}$ ' and $\mathbf{r}$ and needs to be stored (Figure 2).

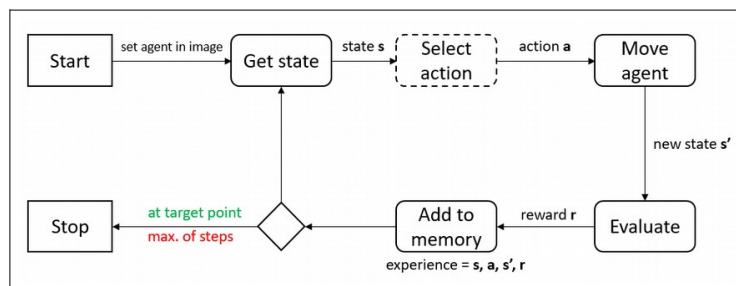

Figure 2: Overview of landmark detection in a medical image using reinforcement learning. The agent will stop after a maximum number of steps or when it has found the target landmark. The `select action' box in the figure is annotated with a dashed line because this is where the action-selection strategies will occur.

In this study, the environment was a cardiac CT image with multiple resolutions (1.0, 2.0, 3.0 and 5.0 $\mathrm{mm}$ ). The agent walked around in the CT image with resolution $1.0 \mathrm{~mm}$ because the reward was computed on this position. The states in the other resolutions were obtained by translating the current position to those resolutions. By using multiple resolutions, the agent had additional information about its current position while preserving the dimensions of the agent's state (an 8x8x8 voxel cube). Additionally, the agent could choose from 26 actions. This action-space covered all the possible single-step directions in a 3D grid (except standing still). Both studies (Ghesu, 2017b) and (Alansary,
2019), described an action-space of six actions and state sizes of $25 \times 25 \times 25$ voxels and $45 \times 45 \times 45$ voxels. After each move, the experience of the agent was stored in prioritized experience replay memory (Schaul, 2015). This type of memory enabled the optimization process to train with experiences which had a higher priority (or learning value) and resulted in faster convergence

When the agent selects an action, it needs to know which action will maximize the cumulative reward over time or, to put it in other terms; the agent needs to know policy $\pi$. This policy defines the agent's behaviour and maps state to actions. The optimal policy knows for every state which action to choose in order to maximize the cumulative reward.

\subsection{Landmark detection with deep Q-learning}

Since it is not possible to map all possible states from cardiac CT to actions, a function approximator is required. In our study, we used a convolutional neural network $(\mathrm{CNN})$ to approach the optimal policy by learning the action-value function $\mathrm{Q}(\mathrm{s}, \mathrm{a})$ (Mnih, 2015). This has already been performed in studies where a deep CNN or deep Q-network (DQN) was trained with the experiences of the agent in order to learn this action-value function Q (Ghesu, 2017b; Alansary, 2019).

In our work, a double duelling DQN method was used to learn this $\mathrm{Q}$ function. Two identical models were used to reduce the overestimation of the Qvalues. Before each training session, the weights of the first model were randomly initialized (Saxe, 2013) and copied to the second model. While the first DQN model was used to retrieve the actions of the current observations, a second (frozen) DQN model yielded Q-values during the optimization process (Van Hasselt, 2016). The weights of the first DQN were transferred to the frozen DQN after a variable number of iterations $(\tau)$.

In order to learn which states contained a higher learning value regardless of the effect of the action (Wang, 2015), an advantage and a value module were introduced in the architecture (Figure 3). 


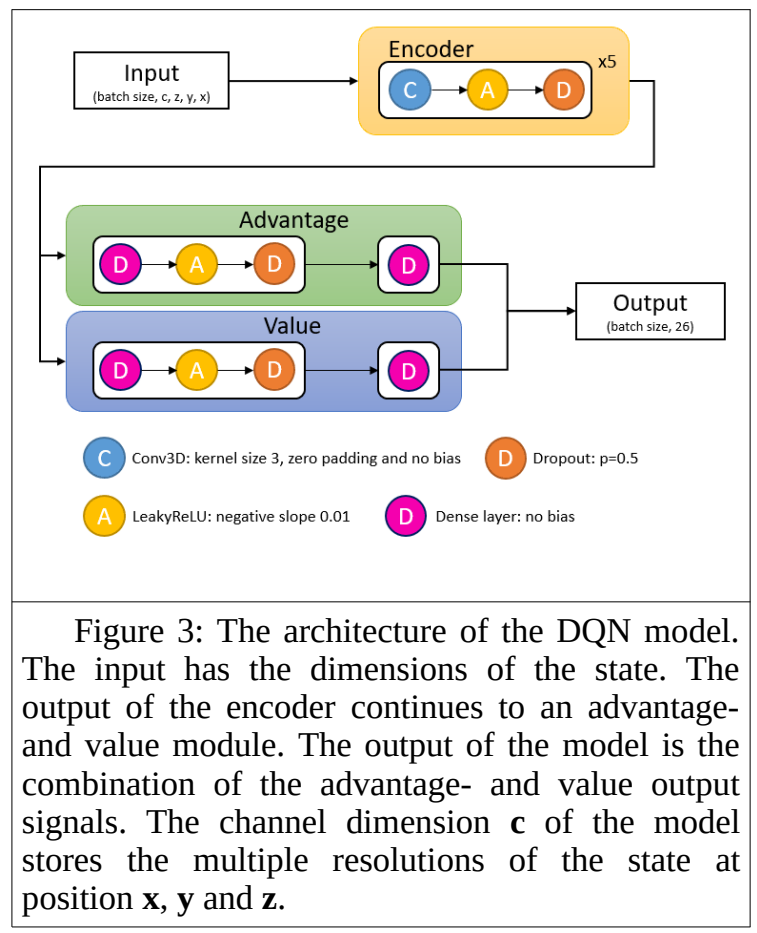

Teaching a DQN to learn this Q function with the experiences of the agent requires iterative trialand-error. Based on its state, the agent chooses an action which is evaluated by measuring the reward. Next, the DQN is updated by using the Bellman equation (Bellman, 1954). This equation returns the Q-value or the maximum future reward, which is the immediate reward for a given state and action and the maximum future reward for the next state.

The size of the output of the DQN model is equal to the size of the action-space. The model's output yields the Q-values for each action for the current state. In order to select the right action for a current state, the index of the highest Q-value is selected, which yields the maximum future reward for the agent.

Initially, the agent selects random actions and the established $\varepsilon$-decay strategy usually guides this random process; however, other strategies were explored in this work.

\subsection{The exploring agent}

To learn the pathway towards the target point, the agent needs to explore and exploit paths. The exploration is required at the beginning of the training to discover new directions towards the target point. The exploitation is required near the end of the training to establish discovered routes. In this study, we compared three strategies to balance exploration and exploitation: the established exponential $\varepsilon$-decay and two novel strategies: $\alpha$ decay and action-dropout.

Exponential $\varepsilon$-decay uses a value $\varepsilon$ that drives the decision for predicting an action or randomly selecting an action. An $\varepsilon$ value needs to be decayed from 0.999 to 0.05 during training. When a random value is smaller than the current $\varepsilon$ value, random action is chosen. Otherwise, the model predicts the Q-values from the current state and the action with the highest Q-value is selected as the predicted action (Figure 4).

Linear $\alpha$-decay always uses the model to predict the Q-values from the current state of the agent. An $\alpha$ value needs to be linearly decayed from 1.0 to 0.0 during training. If a random value is smaller than the current $\alpha$ value, the action with the highest Q-value is ignored, and a random action between the remaining 25 actions is selected. When applied, this strategy ignores the highest Q-value on purpose, causing the agent to find other routes to the target landmark. Otherwise, the action with the highest Qvalue is selected as the predicted action (Figure 4).

Similar to $\alpha$-decay, action-dropout always uses the model to predict the Q-values from the current state of the agent. A threshold value needs to be linearly decayed from 1.0 to 0.0 during training. If a random value is smaller than the current threshold value, action-dropout is applied, which implies that a percentage $(p=0.5)$ of actions are removed from the action-space, and a random action is selected from the reduced action-space. If action-dropout is not applied, the action with the highest Q-value is selected (Figure 4).

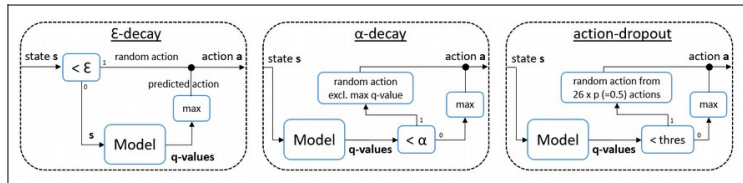

Figure 4: Overview of the three action-selection strategies: $\varepsilon$-decay, $\alpha$-decay and action-dropout. 
In this study, we aimed to train a DQN model with a larger action-space, a smaller state size and less computational resources than the state-of-theart. In order to overcome these challenges, we used curriculum learning.

\subsection{Landmark detection with curriculum deep Q-learning}

Curriculum learning (Bengio, 2009) is inspired by the human education system, where students start with a straightforward concept before learning more advanced concepts. This multiple-concept approach was already applied to object detection, where straightforward networks were trained first (Zhang, 2016). In this study, curriculum learning was applied to the linear distance between starting point $S$ (the center of the aortic root) and target point $\mathrm{T}$ (one of the three landmarks: MS1, MS2 or MS3). The distance between these points was split into ten substarting points. The first and most straightforward concept was defined as the path from the closest sub-starting point $s^{1}$ to $T$. When the agent had learned this path, the next starting points were processed until the model had learned the longest path from $\mathrm{S}$ to $\mathrm{T}$ (Figure 5).

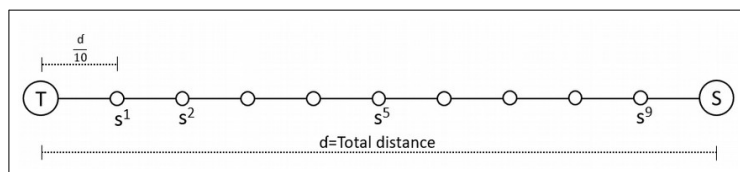

Figure 5: Graphical representation of the curriculum learning approach. The final goal was to learn an entire pathway from the starting point $S$ to the target point $\mathrm{T}$.

\subsection{Training}

In order to train the models, the optimizer Adam (Kingma, 2014) was used with a learning rate of $0.5 \mathrm{e}-5$. This optimizer was chosen to include adaptive learning rates for different parameters. No learning rate decay was introduced since the initial learning rate was set to a small value.

Each time the agent was positioned at a substarting position, random uniform noise was added to this position, which ensured a certain degree of variation in order to make the agent more robust.

\subsection{Validation}

The cohort size was relatively small (278 patients), and k-folding cross-validation was used to validate the method. K-folding $(\mathrm{k}=4)$ divides the entire dataset randomly (with a fixed seed) into four sub-sets, and the training and validation process is repeated four times or folds. Each fold uses a different sub-set as the validation dataset, and the remaining three subsets are used as the training dataset. K-folding enables the usage of the entire dataset as the validation dataset while preserving the separation between training and validation dataset.

The validation process was similar to the training process except that the threshold value (used in the action-selection strategy) was set to -1 . This value forced the agent to use the model to predict the action from the current state.

\subsection{Statistical Analysis}

The accuracy of the landmark detection was assessed by measuring the Euclidean distance from the predicted landmark to the ground truth landmark. All variables were reported as median [lower quartile (LQ) - upper quartile (UQ)]. The agreement between ground truth and predicted landmarks were evaluated using the non-parametric signed Wilcoxon test (with a significant p-value $<0.05$ ).

\subsection{Implementation}

All the computational work was performed on a multi-core computer with a Titan X GPU (NVIDIA Corporation, Los Alamitos, CA). The models and deep learning pipeline were developed with PyTorch v0.4.1 (Paszke, 2014).

\section{RESULTS}

In this section, we discuss the results of the validation process, which were obtained by using the trained models to analyse previously unseen data. The validation process was applied to all three action-selection strategies. Cross-validation was applied to train and validate the method for all three target landmarks: MS1, MS2 and MS3. We obtained 
278 validated patients per target landmark as a result of using k-folding. The action-selection strategies were compared to each other by grouping the results of the three target landmarks into 834 validated patients per strategy. A total of 24 models were trained in this study (each training session used two models), and 12 trained models were used to validate one action-selection strategy.

At the end of the curriculum learning algorithm, the final path from starting point $\mathrm{S}$ to target point $\mathrm{T}$ was learned. The Euclidean distance from the agent's final position to the target point was measured and was considered the predicted landmark of the method. The $\varepsilon$-decay and action-dropout strategy yielded similar results, whereas the $\alpha$-decay strategy was slightly more accurate than the other strategies. The prediction time of the full path for a single patient was below 1 second (Table 1 ).

\begin{tabular}{|c|c|c|c|}
\hline & \multicolumn{3}{|c|}{ Distance from target landmark [mm] } \\
\hline & $\boldsymbol{\varepsilon}$-decay & o-decay & $\begin{array}{c}\text { action- } \\
\text { dropout }\end{array}$ \\
\hline MS1 & $2.4[0.0-4.9]$ & $1.2[0.0-4.2]$ & $2.3[0.0-4.5]$ \\
\hline MS2 & $3.0[0.0-4.7]$ & $0.0[0.0-4.6]$ & $2.0[0.0-4.2]$ \\
\hline MS3 & $2.2[0.0-4.5]$ & $1.4[0.0-4.3]$ & $1.0[0.0-4.2]$ \\
\hline All & $2.4[0.0-4.6]$ & $0.0[0.0-4.4]$ & $2.0[0.0-4.4]$ \\
\hline \multicolumn{4}{|c|}{ Table 1: A summary of the Euclidean distances } \\
from the predicted landmark to the target landmark \\
for all three action-selection strategies. Distances \\
are reported as median [LQ - UQ]. \\
\hline
\end{tabular}

The agent's performance during the curriculum learning process was measured by validating the models before moving to the next sub-starting point. This inter-algorithmic-validation resulted in Euclidean distances between the predicted points and $\mathrm{T}$ for each of the ten sub-starting points. In order to compare action-selection strategies, the distances for each starting point were grouped per strategy. The distances to the target landmarks reduced overtime for all three strategies. The $\alpha$-decay strategy is similar to the other strategies until the final starting point $\mathrm{S}$, where the median distance to the target landmark was zero (Figure 6).

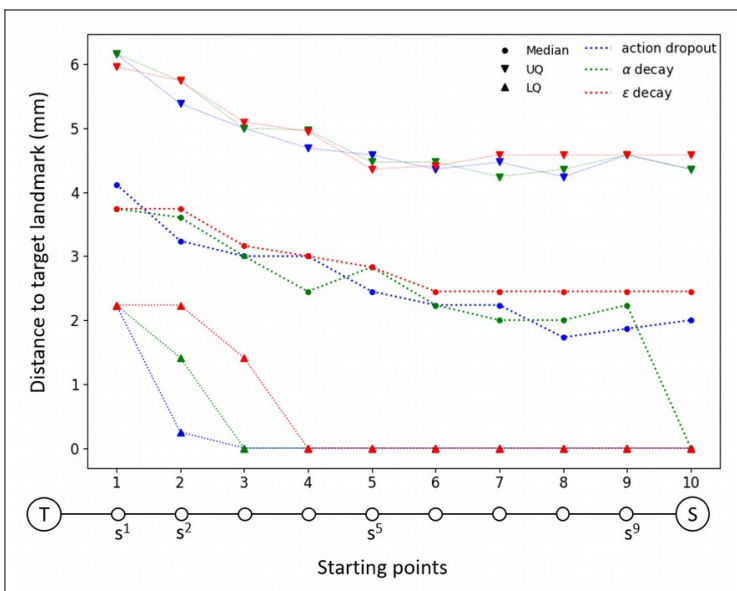

Figure 6: The evolution of the distances to the target landmarks for each sub-starting point for all three action-selection strategies.

An inter-observer variability study was conducted on 20 patients to assess the accuracy of the method. The Euclidean distances between the landmarks annotated by the two observers were used as the difference between both observers. These differences were compared to the predicted landmarks of the $\alpha$-decay strategy of the same 20 patients. The differences between the predictions of the model and observer 1 were overall lower than the differences between observer 1 and 2 (Table 2).

\begin{tabular}{|c|c|c|c|c|}
\hline & $\begin{array}{l}\alpha \text {-decay vs } \\
\text { observer } 1 \\
\left(d_{1}\right)[\mathrm{mm}]\end{array}$ & $\begin{array}{l}\text { Observer } 1 \\
\text { vs observer } \\
2\left(\mathrm{~d}_{2}\right)[\mathrm{mm}]\end{array}$ & $\begin{array}{l}\text { Paired } \\
\text { diff. }\left(d_{2^{-}}\right. \\
\left.\quad d_{2}\right)\end{array}$ & $\mathbf{p}$ \\
\hline MS1 & $2.7[0.0-5.1]$ & $3.8[2.8-5.6]$ & $0.2 \pm 7.9$ & 0.1 \\
\hline MS2 & $0.0[0.0-4.5]$ & $3.4[1.8-4.4]$ & $-1.3 \pm 2.1$ & 0.01 \\
\hline MS3 & $3.0[0.0-4.3]$ & 2.3 [1.4-4.8] & $-0.1 \pm 3.0$ & 1.0 \\
\hline All & $2.6[0.0-4.5]$ & $3.1[1.6-4.9]$ & $-0.4 \pm 5.1$ & 0.4 \\
\hline \multicolumn{5}{|c|}{$\begin{array}{l}\text { Table 2: Comparison of the Euclidean distances } \\
\text { between the predicted and target landmarks }\left(\mathrm{d}_{1}\right) \text {, } \\
\text { and the target points identified by the two } \\
\text { observers }\left(\mathrm{d}_{2}\right) \text {. }\end{array}$} \\
\hline
\end{tabular}

\subsection{Q-landscapes}

In order to provide additional insight into the agent's world view, we plotted Q-landscapes which show the highest Q-values for the entire image (Figure 7). 


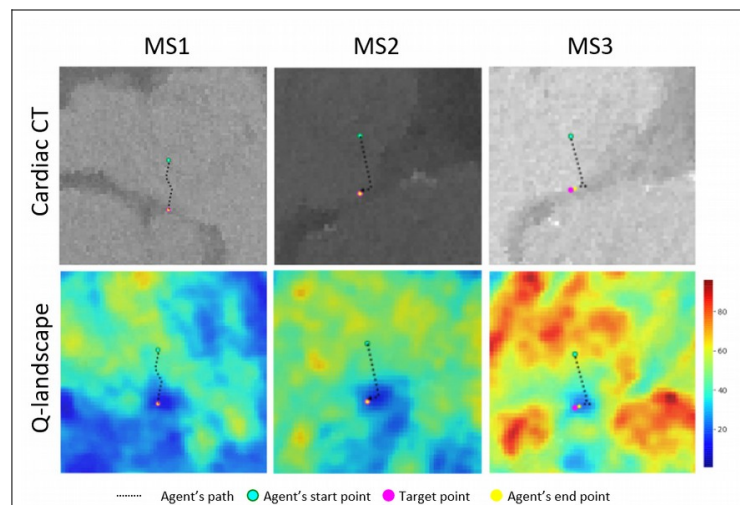

Figure 7: The path of the agent in the cardiac CT image (top) and the Q-landscape (bottom) for each of the target landmarks: MS1, MS2 and MS3. The Q-values are depicted in a heatmap (red are high and blue are low values)

The Q-landscapes were generated by convolving a state-sized cube over the entire image and selecting the highest Q-value. This operation produced a volumetric Q-landscape where each voxel represented the highest Q-value of that particular position. In order to depict the 3D path of the agent, the path was projected on a plane. This plane was defined by the starting point, the target point and the center of those two. Low Q-values can be observed around the target landmarks.

\subsection{Multiple resolutions}

The effect of using multiple resolutions was measured on the $\varepsilon$-decay strategy. This strategy was chosen because it is an already established strategy. In order to measure the effect, three experiments were conducted, each time repeating the entire cross-validation process. The first experiment contained only one resolution $(1.0 \mathrm{~mm})$, the second experiment contained two resolutions (1.0 and 2.0 $\mathrm{mm}$ ), and the final experiment contained all available resolutions. The distances from the predicted landmarks to the target landmarks are summarised in Figure 8. From this plot, it is clear that the first experiment is the least accurate and that the accuracy improves when the number of resolutions increases. This trend clearly shows that the usage of multiple resolutions improves the accuracy of the method.

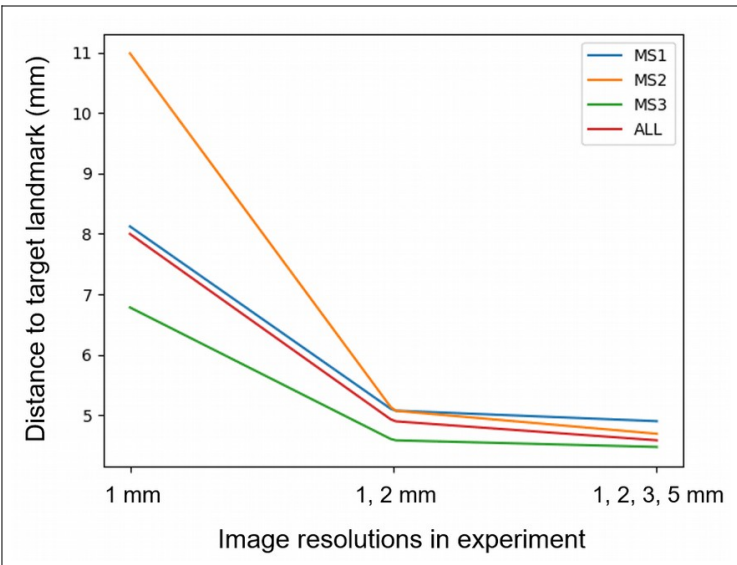

Figure 8: Comparison of the Euclidean distances between the predicted and target landmarks of experiments with different resolutions. Only the upper quartile values are plotted for brevity reasons.

\section{DISCUSSION}

In this feasibility study, a curriculum deep Qlearning method was proposed to detect the inferior border of the membranous septum. We compared three action-selection strategies: $\varepsilon$-decay, $\alpha$-decay and action-dropout, and it was shown that $\alpha$-decay yielded slightly more accurate results. The decaying nature of this strategy can explain this: $\alpha$-decay, when applied, deliberately omits the best action and chooses a random action from the remaining actionspace. The other strategies, when applied, choose a random action from the (reduced) action-space, which may include the best action. The method was efficient since the prediction time per patient was below 1 second. The inter-observer variability study confirmed the accuracy of the method.

Previous studies (Ghesu, 2016; Ghesu, 2017b; Alansary, 2019) presented accurate and efficient landmark detection methods from medical images using deep Q-learning. The authors used clusters of computational resources. In this study, the computational resources were limited to a single graphics processing unit (GPU). Additionally, these studies used larger state sizes and smaller actionspaces. In this study, a state size of $8 \times 8 \times 8$ voxels and 26 actions were used, which is, to the best of our knowledge, the first time such a small state size and 
large action-space was used to detect landmarks from volumetric (medical) images. We expected that a smaller state and a larger action-space would prolong or hamper with the convergence of the models. It is our opinion that the incremental character of curriculum learning is responsible for this speed-up.

Although this preliminary study has proven to be promising, there are a few limitations to be noted. First, the model only learns the path from the center of the aortic annular plane (with small random oscillations) to the target landmarks. When the agent enters areas outside the trained area or discovers image artefacts, it can get lost. This limitation will be handled in future work, wherein the agent will be put on the surface of a sphere around the target landmark with a gradually increasing radius. Next, the cohort size was relatively small, which prevented the introduction of an independent test-dataset. Adding more patients to the cohort can overcome this limitation and will increase the robustness of the method. This limitation can partially be addressed by applying a proper data-augmentation strategy.

There are a couple of recommendations for future work. First, a halting signal should be included. This mechanism can be obtained by adding a stopping action or measure oscillations in Q-values (as described in (Ghesu, 2017b; Alansary, 2019)). Next, searching for landmarks by using multiple agents could be considered (Mnih, 2016; Espeholt, 2018). Finally, the task of detecting landmarks with curriculum deep Q-learning in volumetric images is computationally cheap yet difficult enough to serve as a baseline to experiment with novel deep reinforcement learning techniques.

\section{CONCLUSION}

In conclusion, curriculum deep Q-learning was applied to detect the inferior border of the membranous septum. The output of this study is four-fold: first, the predicted landmarks were detected accurately and efficiently. Next, we have proposed and validated two novel action-selection strategies: $\alpha$-decay and action-dropout and compared it to the already established $\varepsilon$-decay strategy. Thirdly, the agent was equipped with a small state size and burdened with a large action-space yet still managed to detect landmarks accurately. Finally, the incremental character of curriculum learning enabled the research into novel mechanisms and strategies in the domain of deep reinforcement learning.

\section{ACKNOWLEDGEMENTS}

The authors would like to thank both operators that detected the landmarks from cardiac CT for both ground truth and inter-operator data. All computational work was performed on NVIDIA GPUs from the NVIDIA GPU Grant Program. This work was supported by the European Commission within the Horizon 2020 Framework through the MSCA-ITN-ETN European Training Networks (project number 642458).

\section{REFERENCES}

Smith, C. R., Leon, M. B., Mack, M. J., Miller, D. C., Moses, J. W., Svensson, L. G., ... \& Williams, M. (2011). Transcatheter versus surgical aortic-valve replacement in high-risk patients. New England Journal of Medicine, 364(23), 2187-2198.

Leon, M. B., Smith, C. R., Mack, M. J., Makkar, R. R., Svensson, L. G., Kodali, S. K., ... \& Doshi, D. (2016). Transcatheter or surgical aortic-valve replacement in intermediate-risk patients. New England Journal of Medicine, 374(17), 1609-1620.

Reardon, M. J., Van Mieghem, N. M., Popma, J. J., Kleiman, N. S., Søndergaard, L., Mumtaz, M., ... \& Chetcuti, S. (2017). Surgical or transcatheter aorticvalve replacement in intermediate-risk patients. New England journal of medicine, 376(14), 1321-1331.

Popma, J. J., Deeb, G. M., Yakubov, S. J., Mumtaz, M., Gada, H., O’Hair, D., ... \& Askew, J. (2019). Transcatheter aortic-valve replacement with a selfexpanding valve in low-risk patients. New England Journal of Medicine, 380(18), 1706-1715.

Mack, M. J., Leon, M. B., Thourani, V. H., Makkar, R., Kodali, S. K., Russo, M., ... \& Leipsic, J. (2019). Transcatheter aortic-valve replacement with a balloonexpandable valve in low-risk patients. New England Journal of Medicine, 380(18), 1695-1705.

Rocatello, G., El Faquir, N., De Santis, G., Iannaccone, F., Bosmans, J., De Backer, O., ... \& Mortier, P. (2018). Patient-specific computer simulation to elucidate the role of contact pressure in the development of new conduction abnormalities after catheter-based 
implantation of a self-expanding aortic valve. Circulation: Cardiovascular Interventions, 11(2), e005344

Hamdan, A., Guetta, V., Klempfner, R., Konen, E., Raanani, E., Glikson, M., ... \& Spiegelstein, D. (2015). Inverse relationship between membranous septal length and the risk of atrioventricular block in patients undergoing transcatheter aortic valve implantation. JACC: Cardiovascular Interventions, 8(9), 1218-1228.

Zheng, Y., Liu, D., Georgescu, B., Nguyen, H., \& Comaniciu, D. (2015, October). 3D deep learning for efficient and robust landmark detection in volumetric data. In International Conference on Medical Image Computing and Computer-Assisted Intervention (pp. 565-572). Springer, Cham.

Zheng, Y., John, M., Liao, R., Nottling, A., Boese, J., Kempfert, J., ... \& Comaniciu, D. (2012). Automatic aorta segmentation and valve landmark detection in Carm CT for transcatheter aortic valve implantation. IEEE transactions on medical imaging, 31(12), 23072321.

Zhang, J., Liu, M., \& Shen, D. (2017). Detecting anatomical landmarks from limited medical imaging data using two-stage task-oriented deep neural networks. IEEE Transactions on Image Processing, 26(10), 4753-4764.

Payer, C., Štern, D., Bischof, H., \& Urschler, M. (2016, October). Regressing heatmaps for multiple landmark localization using CNNs. In International Conference on Medical Image Computing and Computer-Assisted Intervention (pp. 230-238). Springer, Cham.

O'Neil, A. Q., Kascenas, A., Henry, J., Wyeth, D., Shepherd, M., Beveridge, E., ... \& Poole, I. (2018). Attaining human-level performance with atlas location autocontext for anatomical landmark detection in 3D CT data. In Proceedings of the European Conference on Computer Vision (ECCV) (pp. 0-0).

Al, W. A., Jung, H. Y., Yun, I. D., Jang, Y., Park, H. B., \& Chang, H. J. (2018). Automatic aortic valve landmark localization in coronary CT angiography using colonial walk. PloS one, 13(7), e0200317.

Ghesu, F. C., Georgescu, B., Mansi, T., Neumann, D., Hornegger, J., \& Comaniciu, D. (2016, October). An artificial agent for anatomical landmark detection in medical images. In International Conference on Medical Image Computing and Computer-Assisted Intervention (pp. 229-237). Springer, Cham.

Ghesu, F. C., Georgescu, B., Grbic, S., Maier, A. K., Hornegger, J., \& Comaniciu, D. (2017, September). Robust multi-scale anatomical landmark detection in incomplete 3D-CT data. In International Conference on Medical Image Computing and Computer-Assisted Intervention (pp. 194-202). Springer, Cham.

Ghesu, F. C., Georgescu, B., Zheng, Y., Grbic, S., Maier, A., Hornegger, J., \& Comaniciu, D. (2017). Multiscale deep reinforcement learning for real-time 3Dlandmark detection in CT scans. IEEE transactions on pattern analysis and machine intelligence, 41(1), 176189.
Alansary, A., Oktay, O., Li, Y., Le Folgoc, L., Hou, B., Vaillant, G., ... \& Rueckert, D. (2019). Evaluating reinforcement learning agents for anatomical landmark detection. Medical image analysis, 53, 156-164.

Litjens, G., Kooi, T., Bejnordi, B. E., Setio, A. A. A., Ciompi, F., Ghafoorian, M., ... \& Sánchez, C. I. (2017). A survey on deep learning in medical image analysis. Medical image analysis, 42, 60-88.

Sahiner, B., Pezeshk, A., Hadjiiski, L. M., Wang, X., Drukker, K., Cha, K. H., ... \& Giger, M. L. (2019). Deep learning in medical imaging and radiation therapy. Medical physics, 46(1), e1-e36.

Schaul, T., Quan, J., Antonoglou, I., \& Silver, D. (2015). Prioritized experience replay. arXiv preprint arXiv:1511.05952.

Mnih, V., Kavukcuoglu, K., Silver, D., Rusu, A. A., Veness, J., Bellemare, M. G., ... \& Petersen, S. (2015). Human-level control through deep reinforcement learning. Nature, 518(7540), 529.

Van Hasselt, H., Guez, A., \& Silver, D. (2016, March). Deep reinforcement learning with double q-learning. In Thirtieth AAAI conference on artificial intelligence.

Wang, Z., Schaul, T., Hessel, M., Van Hasselt, H., Lanctot, M., \& De Freitas, N. (2015). Dueling network architectures for deep reinforcement learning. arXiv preprint arXiv:1511.06581.

Bengio, Y., Louradour, J., Collobert, R., \& Weston, J. (2009, June). Curriculum learning. In Proceedings of the 26th annual international conference on machine learning (pp. 41-48). ACM.

Zhang, K., Zhang, Z., Li, Z., \& Qiao, Y. (2016). Joint face detection and alignment using multitask cascaded convolutional networks. IEEE Signal Processing Letters, 23(10), 1499-1503.

Kingma, D. P., \& Ba, J. (2014). Adam: A method for stochastic optimization. arXiv preprint arXiv:1412.6980.

Saxe, A. M., McClelland, J. L., \& Ganguli, S. (2013). Exact solutions to the nonlinear dynamics of learning in deep linear neural networks. arXiv preprint arXiv:1312.6120.

Paszke, A., Gross, S., Chintala, S., Chanan, G., Yang, E., DeVito, Z., ... \& Lerer, A. (2017). Automatic differentiation in pytorch.

Mnih, V., Badia, A. P., Mirza, M., Graves, A., Lillicrap, T., Harley, T., ... \& Kavukcuoglu, K. (2016, June). Asynchronous methods for deep reinforcement learning. In International conference on machine learning (pp. 1928-1937).

Espeholt, L., Soyer, H., Munos, R., Simonyan, K., Mnih, V., Ward, T., ... \& Legg, S. (2018). Impala: Scalable distributed deep-rl with importance weighted actorlearner architectures. arXiv preprint arXiv:1802.01561.

Bellman, R. (1954). The theory of dynamic programming. Bulletin of the American Mathematical Society, 60(6), 503-515. 


\section{APPENDIX}

\section{Algorithmic details}

The pseudo-code of the used curriculum deep Qlearning algorithm is presented in Algorithm 1. The model learns the paths from starting point $s^{1}$ to $s^{10}$ or S. In this section; we explain a few parameters. The maximum number of steps that the agent could make was initially set to 15 to avoid the agent getting lost at the beginning of the algorithm. The algorithm gradually increased the maximum number of steps to ensure that the agent had enough time to reach the target point. The number of iterations $\tau$ triggered the weight transfer from the first DQN to the frozen DQN and was initially set to a small value. Therefore, numerous patients would pass before the weight transfer. We observed that transferring the weights too quickly resulted in models which were not able to find the target point. The algorithm increased the parameter $\tau$ when the starting points were set further away from the target points.

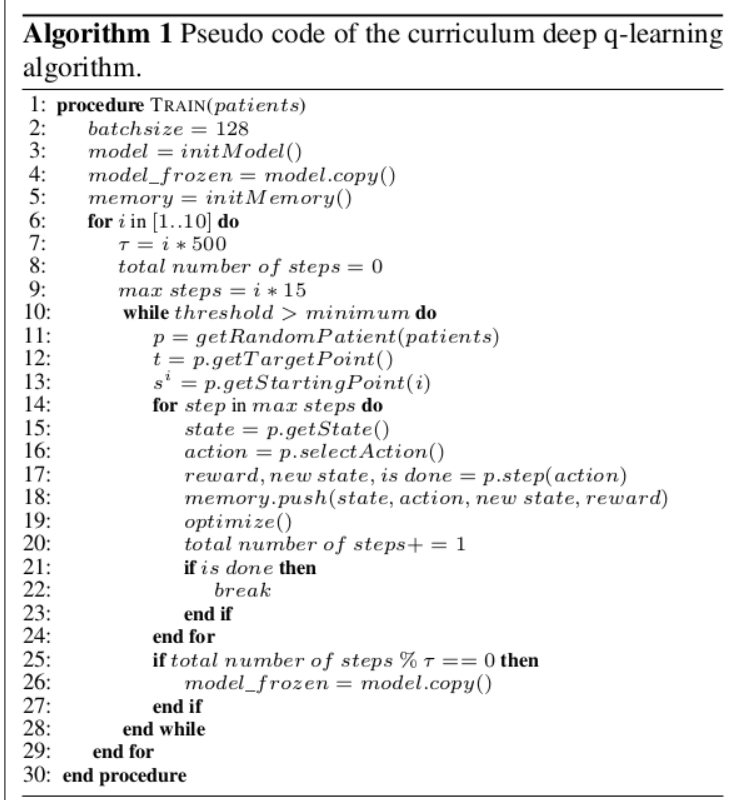

Figure 1: Pseudo code of the curriculum deep Q-learning algorithm. 